\title{
OPTIMAL SOLUTION OF A DIFFUSION EQUATION WITH A DISCRETE SOURCE TERM
}

\author{
A. ARAÚJO, MARIA F. PATRÍCIO AND JOSÉ L. SANTOS
}

\begin{abstract}
In this paper we study the numerical behavior of a diffusion equation with a discrete control source term. The equation is discretized in space by finite differences and in time by an implicit scheme. The control variables are calculated in order to minimize an objective function, taking into account some restrictions. We define two strategies to obtain the optimal solution and present some numerical results in a context of a model that describes the oxygen concentration in a single chamber microbial fuel cell.
\end{abstract}

KEYWORDS: diffusion equation, finite differences, quadratic optimization problem. AMS SubJeCt Classification (2000): 65M06, 90C20.

\section{Introduction}

The purpose of this work is to study the numerical behavior of a diffusion equation with a discrete source term and control variables. We will consider a discretization of the partial differential equation and use optimization techniques in order to obtain a numerical solution that minimizes a given objective function.

The paper is organized as follows. In Section 2, we describe the problem that we want to solve and, in Section 3, the numerical method used in the discretization. At the end of this section we prove a result that indicates a qualitative behavior of the numerical solution. In Section 4, we characterize the optimal solution using two different approaches, with and without time dependence. In Section 5, we apply our procedure to a model that describes the oxygen concentration in a single chamber microbial fuel cell [2], [6]. The idea is to obtain the optimal cost pumping of a fixed amount of oxygen by choosing the source points. We prove that, in order to minimize the cost function, the pumping must be done at the beginning of the chamber and, when the sparging rate is time dependent, late in time.

Received November 14, 2007. 


\section{The problem}

Let us consider a diffusion equation with a discrete source term in the form

$$
\left.\left.u_{t}=\alpha u_{x x}+\sum_{i=1}^{r} q_{v_{i}}(t) \delta\left(x-y_{i}\right), \quad x \in\right] 0, L[, t \in] 0, T\right],
$$

where $L$ and $T$ are two positive real constants and $\delta$ is the Dirac delta function. The dependent variable $u$ could be the concentration of a given substance, $\alpha>0$ its diffusion coefficient and $r$ the number of points where we consider the "injections". For $i \in\{1, \ldots, r\}, q_{v_{i}}(t)$ is a control variable which gives the measure of the "sparging rate" of the substance in the point $\left.y_{i} \in\right] 0, L[$, in the instance $t$.

Let us now consider the boundary conditions

$$
\left.\left.u(0, t)=c_{0} \quad \text { and } \quad \frac{\partial u}{\partial x}(L, t)=0, \quad t \in\right] 0, T\right],
$$

as well as the initial condition

$$
u(x, 0)=0, \quad x \in] 0, L[.
$$

The control variables are calculated by solving an optimization problem. In this work, we consider the problem presented in [6], which is described in the following way:

$$
\begin{aligned}
& \min f(Q)=C \sum_{i=1}^{r} \int_{0}^{T}\left(u_{b}-u\left(y_{i}, t\right)\right) q_{v_{i}}(t) d t, \\
& \text { s.t. : } \\
& \qquad \sum_{i=1}^{r} \int_{0}^{T} q_{v_{i}}(t) d t=K, \\
& q_{v_{i}}(t) \geq 0, \quad i \in\{1, \ldots, r\}, t \in[0, T],
\end{aligned}
$$

where $K$ and $C$ are positive constants, and $u_{b}$ is an upper bound for the concentration.

In the next section we will consider a discrete version of this problem. The numerical solution is obtained by solving the discrete problem with optimization techniques. 


\section{Numerical approach}

Let us consider in $[0, L] \times[0, T]$ a grid $\left\{\left(x_{i}, t_{j}\right): i=0, \ldots, n, j=0, \ldots, m\right\}$ with $x_{0}=0, x_{n}=L, t_{0}=0, t_{m}=T$, with constant step sizes $\Delta x=x_{i}-x_{i-1}$, $i=1, \ldots, n$ and $\Delta t=t_{j}-t_{j-1}, j=1, \ldots, m$. Let us also consider $\left\{v_{1}, \ldots, v_{r}\right\} \subseteq\{1, \ldots, n-1\}$ the indexes of the spatial discretization nodes where the injections are consider, i.e., $x_{v_{i}}=y_{i}, 1 \leq i \leq r$. Using the second-order centered finite difference operator in space and the implicit Euler method in time, the solution $u$ of (1)-(3) can be approximated by the solution of the linear systems

$$
M U^{j}=U^{j-1}+R \overline{c_{0}}+\Delta t Q^{j}, \quad j \in\{1, \ldots, m\},
$$

where $U^{j}=\left(u_{1}^{j}, \ldots, u_{n-1}^{j}\right)^{\top}$, with $u_{i}^{j} \approx u\left(x_{i}, t_{j}\right), Q^{j}=\left(q_{1}^{j}, \ldots, q_{n-1}^{j}\right)^{\top}, 1 \leq$ $j \leq m$, with $q_{i}^{j}=0$, for all $i \in\{1, \ldots, n-1\} \backslash\left\{v_{1}, \ldots, v_{r}\right\}$ and all $j \in$ $\{0, \ldots, m\}, \overline{c_{0}}=\left(c_{0}, 0, \ldots, 0\right)^{\top}, M_{(n-1) \times(n-1)}$ a tridiagonal matrix with $m_{i, i}=$ $2 R+1, m_{i, i+1}=m_{i+1, i}=-R(1 \leq i \leq n-2)$ and $m_{(n-1),(n-1)}=1+R$, and $R=\alpha \frac{\Delta t}{\Delta x^{2}}$. The vector $U^{0}$ is the null vector. Note that the control variables are $q_{v_{1}}^{j}, \ldots, q_{v_{r}}^{j}$; the other components of the vector $Q^{j}$ were introduced in order to write the vectorial equation (7).

According to (7), we may write

$$
U^{j}=\Delta t \sum_{k=1}^{j} N^{k} Q^{j+1-k}+N\left(I-N^{j}\right)(I-N)^{-1} R \overline{c_{0}}, \quad j \in\{1, \ldots, m\},
$$

where $N=M^{-1}$.

Let us now consider the following result.

Proposition 1. Assume that $Q^{j}=0$ and $U^{j}$ satisfying (7), for all $j \in$ $\{1, \ldots, m\}$. Then:

- $U^{j}-U^{j-1} \geq 0, \quad \forall j \in\{1, \ldots, s\}$;

- $u_{i-1}^{j} \geq u_{i}^{j}, \quad \forall j \in\{1, \ldots, s-1\}, \forall i \in\{1, \ldots, n\}$.

Proof: Let us first note that the matrix $M$ in (7) is a M-matrix [5]. In fact, $m_{i, i}>0, m_{i, j} \leq 0$, for all $i$ and $j$, and $M$ is strictly diagonal dominant. Then, $N=M^{-1} \geq 0$. We may easily see that $U^{j}-U^{j-1}=N^{j-1}\left(U^{1}-U^{0}\right)$. But, $U^{0}=0$ and $U^{1}=\overline{c_{0}}$ and then, $U^{j}-U^{j-1} \geq 0$, which concludes the first part of the proof.

The second part results from the fact that $D_{-x} U^{j} \leq 0$, where $D_{-x}$ is the first order backward differentiation matrix. In fact, if we consider $Q^{j}=0$, 
we may write the linear system in the form $A\left(U^{j+1}-U^{j}\right)=D_{-x} U^{j+1}$, where $A$ is a negative matrix. Since $U^{j+1}-U^{j} \geq 0$ we may conclude the proof.

A discrete approximation for the optimal solution of (4)-(6) can be obtained by solving the optimization problem

$$
\begin{aligned}
& \min f(Q)=\Delta t C \sum_{j=1}^{m} \sum_{i=1}^{r}\left(u_{b}-u_{v_{i}}^{j-1}\right) q_{v_{i}}^{j}, \\
& \text { s.t. : } \\
& \Delta t \sum_{j=1}^{m} \sum_{i=1}^{r} q_{v_{i}}^{j}=K, \\
& q_{i}^{j} \geq 0, \quad i \in\{1, \ldots, n-1\}, j \in\{1, \ldots, m\} .
\end{aligned}
$$

This strategy could be generalized to other objective functions. Replacing (8) in (9), we obtain a quadratic programming problem and we may use known numerical algorithms to solve it [1], [3], [4].

\section{Optimal solution}

To obtain an approximation to the solution of (1)-(6), we solve (8)-(11) using two different strategies. In a first case we consider $q$, in (1), as a time independent function. Then the goal is to know where and how the substance must be injected in the system. In a second case, we consider $q$ as a time dependent function and so the purpose is to know where, when e and how the substance must be injected in the system.

We now describe the optimal solutions for the two different strategies described above.

4.1. Time independent case. If we consider $q$, in (1), as a time independent function, we have, in the discrete equation, $q_{i}^{j}=q_{i}$, for all $i \in$ $\{1, \ldots, n-1\}$ and all $j \in\{1, \ldots, m-1\}$. So, we may write $(8)$ in the form

$$
U^{j}=\Delta t Q \sum_{k=1}^{j} N^{k}+N\left(I-N^{j}\right)(I-N)^{-1} R \overline{c_{0}}, \quad j \in\{1, \ldots, m\},
$$

where $Q=\left[q_{i}\right]$.

Let us now define the matrices $P_{j}=\sum_{k=1}^{j} N^{k}$. These matrices may be written in the form

$$
P_{j}=N\left(I-N^{j}\right)(I-N)^{-1}=\left(I-N^{j}\right)(M-I)^{-1}
$$


and so (12) takes the form

$$
U^{j}=P_{j}\left(\Delta t Q+R c_{0}\right), \quad j \in\{1, \ldots, m\} .
$$

In this case, the discrete optimization problem is

$$
\begin{gathered}
\min f(Q)=\Delta t C Q^{T}\left(U_{b}-\sum_{j=1}^{m} P_{j-1}\left(\Delta t Q+R c_{0}\right)\right), \\
\text { s.t. : } \\
\qquad \sum_{i=1}^{r} q_{v_{i}}=K / T, \\
q_{i} \geq 0, \quad i \in\{1, \ldots, n-1\},
\end{gathered}
$$

where $U_{b}$ is a vector with all entries equal to $u_{b}$.

The following lemma shows that we minimize the total cost if we inject all the substance in the first injection point, i.e., in $y_{1}$.

Lemma 1. Let $Q \geq 0,1 \leq i \leq n-1$, be an admissible solution for the control variables of the problem (14)-(16), i.e.,

$$
q_{i}=0, \quad i \in\{1, \ldots, n-1\} \backslash\left\{v_{1}, \ldots, v_{r}\right\} .
$$

Let us define $\bar{Q}=\left[\bar{q}_{i}\right]$ such that

$$
\bar{q}_{v_{1}}=\sum_{\ell=1}^{r} q_{v_{\ell}} \quad \text { and } \quad \bar{q}_{i}=0, \quad \forall i \neq v_{1} .
$$

Let $\left(p_{j}\right)_{i, k}$ and $\eta_{i, k}$ be the $(i, k)$-entries of the matrices $P_{j}$ and $N$, respectively. If

$$
\frac{c_{0} R}{K}>\gamma:=\frac{n / R-\min _{1 \leq i, k \leq n}\left(\eta_{i, k}\right)}{\min _{1 \leq j \leq m}\left(\left(p_{j}\right)_{v_{1}, 1}-\left(p_{j}\right)_{v_{2}, 1}\right)},
$$

then $f(\bar{Q}) \leq f(Q)$.

Proof: To obtain the expression for the $(i, k)$-entry of $P_{j}$, let us note that the $(i, k)$-entry of $(M-I)^{-1}$ is $\min \{i, k\} / R$. Then

$$
\left(p_{j}\right)_{i, k}=\sum_{\ell=1}^{n}\left(\delta_{i, \ell}-\eta_{i, \ell}^{j}\right) \min \{\ell, k\} / R,
$$


where $\delta_{i, \ell}$ is the Kronecker symbol and $\eta_{i, \ell}^{j}$ the $(i, \ell)$-entry of $N^{j}$. Let us define

$$
P_{\infty}=\lim _{j=\infty} P_{j} .
$$

We may conclude that $P_{\infty}=N(I-N)^{-1}=(M-I)^{-1}$, because

$$
\lim _{j \rightarrow \infty} N^{j}=0 \text {. }
$$

This last equality results from the fact that the eigenvalues of $N$, of the form (see [7])

$$
\left(1+4 R \sin ^{2}\left(\frac{2 i-1}{2 n+1} \frac{\pi}{2}\right)\right)^{-1}, \quad i \in\{1, \ldots, n\},
$$

are all in $] 1 /(4 R+1), 1[$. But $N \geq 0$ (proved in Proposition 1), and so $N \leq P_{j} \leq P_{\infty}$, i.e.,

$$
\eta_{i, k} \leq\left(p_{j}\right)_{i, k} \leq\left(p_{\infty}\right)_{i, k}=\min \{i, k\} / R \leq n / R .
$$

Let $U^{j}$ (respectively, $\bar{U}^{j}$ ) be the vector obtained from $Q$ (respectively, $\bar{Q}$ ) by (13). Then

$$
U^{j}=P_{j}\left(R c_{0}+\Delta t Q\right) \quad \text { and } \quad \bar{U}^{j}=P_{j}\left(R c_{0}+\Delta t \bar{Q}\right) .
$$

If we prove that $\bar{u}_{v_{1}}^{j} \geq u_{v_{i}}^{j}, 1 \leq i \leq r, 1 \leq j \leq m$, then $f(\bar{Q}) \leq f(Q)$ follows immediately. We have

$$
\bar{u}_{v_{1}}^{j}=R c_{0}\left(p_{j}\right)_{v_{1}, 1}+\Delta t\left(p_{j}\right)_{v_{1}, v_{1}} \sum_{\ell=1}^{r} q_{v_{\ell}}
$$

and

$$
u_{v_{i}}^{j}=R c_{0}\left(p_{j}\right)_{v_{i}, 1}+\Delta t \sum_{\ell=1}^{r} q_{v_{\ell}}\left(p_{j}\right)_{v_{i}, v_{\ell}} .
$$

Then,

$$
\bar{u}_{v_{1}}^{j}-u_{v_{i}}^{j}=R c_{0}\left(\left(p_{j}\right)_{v_{1}, 1}-\left(p_{j}\right)_{v_{i}, 1}\right)+\Delta t \sum_{\ell=1}^{r} q_{v_{\ell}}\left(\left(p_{j}\right)_{v_{1}, v_{1}}-\left(p_{j}\right)_{v_{i}, v_{\ell}}\right) .
$$

For $i=1$,

$$
\bar{u}_{v_{1}}^{j}-u_{v_{1}}^{j}=\Delta t \sum_{\ell=1}^{r} q_{v_{\ell}}\left(\left(p_{j}\right)_{v_{1}, v_{1}}-\left(p_{j}\right)_{v_{1}, v_{\ell}}\right) \geq 0
$$


because $\left(p_{j}\right)_{v_{1}, v_{1}}-\left(p_{j}\right)_{v_{1}, v_{\ell}} \geq 0$. For $i \geq 2$, Proposition 1 tells us that $\left(p_{j}\right)_{v_{1}, 1}$, $\ldots,\left(p_{j}\right)_{v_{r}, 1}$ is a monotonically decreasing sequence. Then

$$
\left(p_{j}\right)_{v_{1}, 1}-\left(p_{j}\right)_{v_{i}, 1} \geq\left(p_{j}\right)_{v_{1}, 1}-\left(p_{j}\right)_{v_{2}, 1}>0 \quad(2 \leq i \leq r)
$$

and so

$$
\bar{u}_{v_{1}}^{j}-u_{v_{i}}^{j} \geq 0 \Leftrightarrow R c_{0} \geq \Delta t \sum_{\ell=1}^{r} q_{v_{\ell}} \frac{\left(p_{j}\right)_{v_{i}, v_{\ell}}-\left(p_{j}\right)_{v_{1}, v_{1}}}{\left(p_{j}\right)_{v_{1}, 1}-\left(p_{j}\right)_{v_{i}, 1}}
$$

Once

$$
\left(p_{j}\right)_{v_{i}, v_{\ell}}-\left(p_{j}\right)_{v_{1}, v_{1}} \leq n / R-\min _{1 \leq h, k \leq n} \eta_{h, k}
$$

and

$$
\left(p_{j}\right)_{v_{1}, 1}-\left(p_{j}\right)_{v_{i}, 1} \geq\left(p_{j}\right)_{v_{1}, 1}-\left(p_{j}\right)_{v_{2}, 1} \quad(2 \leq i \leq r),
$$

we conclude that if

$$
\frac{c_{0} R}{K}>\gamma
$$

then $\bar{u}_{v_{1}}^{j}-u_{v_{i}}^{j} \geq 0$, which concludes the proof.

According to this lemma, it is possible to obtain an analytical solution for the problem (14)-(16).

Proposition 2. The optimal solution of (14)-(16) is given by

$$
q_{v_{1}}=K / T \quad \text { and } \quad q_{i}=0, \quad \forall i \in\{1, \ldots, n-1\} \backslash\left\{v_{1}\right\} .
$$

Then the numerical solution obtained by (13) is an approximate solution to the exact solution of (1)-(3) with truncation error $\mathcal{O}\left(\Delta x^{2}, \Delta t\right)$.

Proof: It follows immediately from Lemma 1 and from known results for the finite difference scheme used in the discretization.

We are now in position to present the following result which is a corollary of the previous proposition.

Corollary 1. For the problem (14)-(16), the optimal source location is $v_{1}=$ 1 .

4.2. Time dependent case. For this case we cannot obtain an analytical solution to the optimization problem. Nevertheless, our numerical tests show that the best location to feed the system is still the point $x_{1}$ (i.e., $r=1$ and $v_{1}=1$ ), which agrees with the previous case: only $Q^{1}$ is non null, in spite of the last components of this vector be positive (i.e., the $K$ units of substance available are injected in a short period of time, near to the final time). 


\section{Numerical results}

5.1. Computational aspects. Once the vectors $Q^{j}$ are obtained, we must compute $u_{i}^{j}$ in the given mesh in order to obtain an approximation to the solution of the problem that we want to solve. We may obtain these values using (8), in spite of its computational inefficiency. An alternative approach consists in avoiding the inversion of the matrix $M$ factorizing $M=L D L^{\top}$ and solving the linear systems. This approach is computationally more efficient. In fact, when we compute $U^{j}$ using (8) we need $\mathcal{O}\left(m \times n^{3}\right)$ floating-point operations, while, if we use the factorization of $M$ in (7) we only need $\mathcal{O}(m \times$ $n$ ) operations.

Note that, for the first strategy (time independent $q$ ), the values of $Q$ are obtained without any computational effort (Proposition 2) while for the second one (time dependent $q$ ), the solutions $Q^{j}$ are obtained applying the quadprog routine of the MatLab Optimization Toolbox ${ }^{\mathrm{TM}}$.

5.2. Example. Let us consider the problem (1)-(3), with $\alpha=20, L=$ $310, T=50$ and $c_{0}=40$. This equation may be viewed as a model for the oxygen diffusion in a microbial fuel cell [2], [6]. A microbial fuel cell converts chemical energy, available in a bio-convertible substrate, directly into electricity. To achieve this, bacteria are used as a catalyst to convert substrate into electrons.

We admit that, in (9)-(11), $K=350$ and $u_{b}=50$. In order to compute the numerical solution, we consider the strategies explained before.

5.2.1. Time independent case. We consider, in $[0, L]$, different meshes with $\Delta x \in\{0.125,0.25,0.5,1\}$. In Table 1 we compare two situations that correspond to the case where we consider $r=2$ and $r=5$ sources, equally distributed in the spatial domain. The temporal step sizes considered in the discretization were $\Delta t=0.5$ and $\Delta t=1$. In Table 1 we include the values of $q_{i}, i \in\{1, \ldots, r\}$ that minimizes the pumping costs and, for $t=T$, the percentage of the error given by

$$
100 \frac{\left\|U^{m}-\bar{U}^{m}\right\|}{\left\|\bar{U}^{m}\right\|},
$$

where $\|\cdot\|$ is the euclidian norm and $\bar{U}$ the reference solution. 


\begin{tabular}{cc|ccc|cc}
\multicolumn{1}{c}{ step } & \multicolumn{8}{c}{ optimal pumping } & \multicolumn{2}{c}{ quality of the numerical solution } \\
\multicolumn{1}{c|}{$\Delta t$} & $\Delta x$ & $i$ & 1 & 2 & cost & error $(\%)$ \\
\hline 0.5 & 1 & $q_{i}$ & 7 & 0 & 159791.2 & 5.8477 \\
& 0.5 & $q_{i}$ & 7 & 0 & 166992.8 & 3.5439 \\
& 0.25 & $q_{i}$ & 7 & 0 & 170594.3 & 1.6706 \\
& 0.125 & $q_{i}$ & 7 & 0 & 172395.1 & - \\
\hline 1 & 1 & $q_{i}$ & 7 & 0 & 159940.2 & 5.8478 \\
& 0.5 & $q_{i}$ & 7 & 0 & 167060.1 & 3.5440 \\
& 0.25 & $q_{i}$ & 7 & 0 & 170620.8 & 1.6706 \\
& 0.125 & $q_{i}$ & 7 & 0 & 172401.2 & - \\
\hline & & $v_{i}$ & 100 & 200 & &
\end{tabular}

\begin{tabular}{|c|c|c|c|c|c|c|c|c|c|}
\hline \multicolumn{2}{|c|}{ step } & \multicolumn{6}{|c|}{ optimal pumping } & \multicolumn{2}{|c|}{ quality of the numerical solution } \\
\hline$\Delta t$ & $\Delta x$ & $i$ & 1 & 2 & 3 & 4 & 5 & cost & error $(\%)$ \\
\hline 0.5 & 1 & $q_{i}$ & 7 & 0 & 0 & 0 & 0 & 144671.4 & 5.5700 \\
\hline & 0.5 & $q_{i}$ & 7 & 0 & 0 & 0 & 0 & 151850.4 & 3.3757 \\
\hline & 0.25 & $q_{i}$ & 7 & 0 & 0 & 0 & 0 & 155440.3 & 1.5913 \\
\hline & 0.125 & $q_{i}$ & 7 & 0 & 0 & 0 & 0 & 157235.2 & - \\
\hline 1 & $\overline{1}$ & $q_{i}$ & 7 & 0 & $\overline{0}$ & 0 & 0 & 145035.8 & 5.5666 \\
\hline & 0.5 & $q_{i}$ & 7 & 0 & 0 & 0 & 0 & 152132.2 & 3.3736 \\
\hline & 0.25 & $q_{i}$ & 7 & 0 & 0 & 0 & 0 & 155680.7 & 1.5903 \\
\hline & 0.125 & $q_{i}$ & 7 & 0 & 0 & 0 & 0 & 157455.0 & - \\
\hline
\end{tabular}

TABLE 1. Results for time independent $q$ and $r$ equally distributed source terms in $[0, L]$.

For a better visualization of the behavior of $u(x, t)$ in space, we present, in Figure 1, the value of $u(x, T)$ (considering $\Delta t=1$ ) and for the spatial discretizations with $\Delta x \in\{0.5,1\}$.

From the analysis of these results, we may conclude that, in the optimal case, the injection of the substance is always done in the first point (position $v_{1}$ ). We may also conclude that, increasing the value of $r$ (i.e., putting the injection points closer the origin), we decrease the cost. Decreasing the step size $\Delta t$, the cost decreases slightly. The error is not substantially affected 


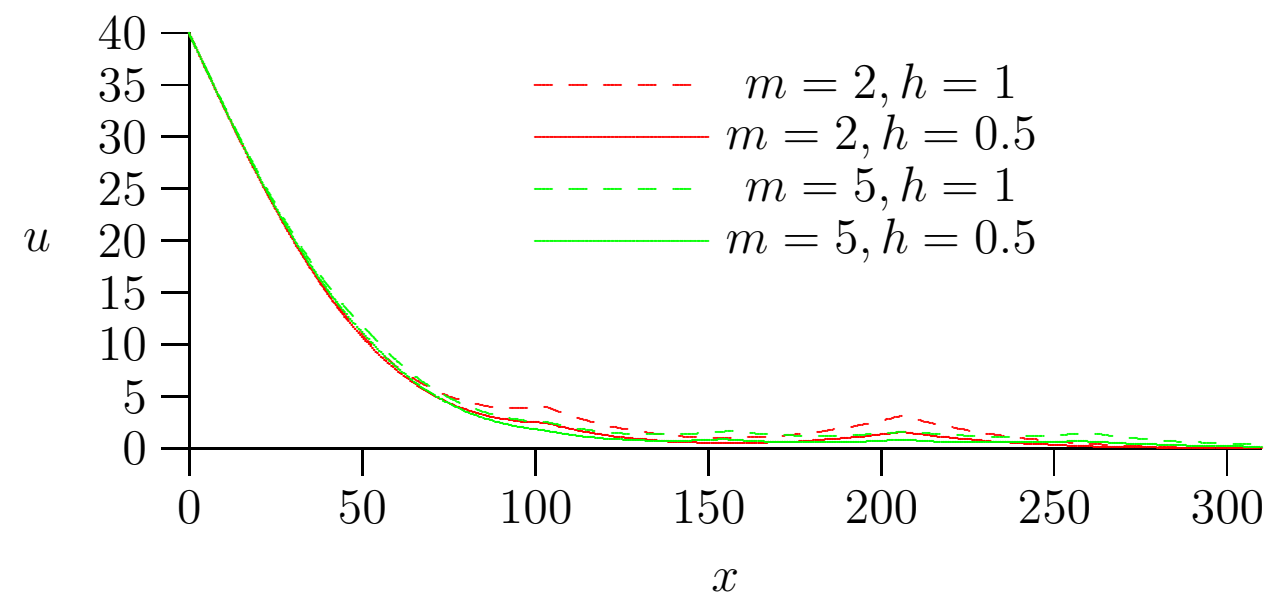

Figure 1. Values of $u$ for different locations $\left(v_{1}\right)$ of the $\operatorname{pump}(\Delta t=1)$.

with $r$ and $\Delta t$. Finally, decreasing the step size $\Delta x$, we improve the quality of the solution (the error decreases faster than $\Delta x$ ) but the cost increases.

The results obtained confirm that the only interesting case is when the injection is done in the position $v_{1}$ (first point available). Then, according to the previously exposed, we force $v_{1}=x_{1}$, independently of the discretization (i.e., $\Delta x$ ). In Table 2 we present the computational results for the same problem but with a different diffusion coefficient $\alpha=86.4$. We also consider $\Delta x=4$ and $\Delta t=0.05$ in order to compare our results with the ones obtained by Zielke [6]. As we may see, our results are in agreement with the ones reported in Zielke's work. In fact, when we increase the diffusion coefficient $\alpha$ the cost and error decrease (except for $\Delta x=4$ where we obtain a worse cost). We may also conclude that the results presented in Table 2 are in concordance with the corresponding ones presented in Table 1 in respect to the variation of $\Delta x$ and $\Delta t$ (except the case $\alpha=20, \Delta x=4$ ). The cost obtained by Zielke [6] with 80 nodes $(\Delta x \approx 4), \Delta t=0.05$ and $\alpha=86.4$ is around 75000 and we obtained a better cost: 40261.74 .

5.2.2. Time dependent case. The previous study was also done for the case where $q$ is time dependent using the technique described above. According to the results presented in Tables 3 and 4, the computational results present similar behavior when compared to the previous case. Note that the cost is reduced and, on the other hand, the percentage of error increased due to a great among of substance is injected late in time. This fact also leads to 
OPTIMAL SOLUTION OF A DIFFUSION EQUATION WITH A DISCRETE SOURCE TERM 11

\begin{tabular}{ccccccccc} 
& & \multicolumn{7}{c}{$\Delta x$} \\
\cline { 3 - 9 }$\alpha$ & $\Delta t$ & & 4 & 2 & 1 & 0.5 & 0.25 & 0.125 \\
\hline \hline & & $v_{1}$ & 4 & 2 & 1 & 0.5 & 0.25 & 0.125 \\
& & $n$ & 77 & 155 & 310 & 620 & 1240 & 2480 \\
\hline 20 & 0.5 & cost & 38379.1 & 41537.8 & 40124.6 & 38566.9 & 37561.6 & 37000.6 \\
& & error (\%) & 2.3493 & 0.8491 & 0.3003 & 0.1017 & 0.0289 & - \\
\cline { 3 - 9 } & 1.0 & cost & 39831.7 & 42921.6 & 41505.1 & 39954.1 & 38954.5 & 38396.8 \\
& & error (\%) & 2.3490 & 0.8490 & 0.3003 & 0.1017 & 0.0289 & - \\
\hline 86.4 & 0.05 & cost & 40261.4 & 38784.7 & 37247.4 & 36266.7 & 35721.9 & 35435.6 \\
& & error (\%) & 0.5586 & 0.1992 & 0.0700 & 0.0236 & 0.0067 & - \\
\cline { 3 - 9 } & 0.5 & cost & 41506.3 & 40027.6 & 38496.3 & 37520.6 & 36978.6 & 36693.9 \\
& & error (\%) & 0.5586 & 0.1992 & 0.0700 & 0.0236 & 0.0067 & - \\
\cline { 2 - 8 } & 1.0 & cost & 42889.1 & 41408.4 & 39884.0 & 38913.7 & 38375.0 & 38092.1 \\
& & error (\%) & 0.5586 & 0.1992 & 0.0700 & 0.0237 & 0.0067 & - \\
\hline
\end{tabular}

TABLE 2. Results for $v_{1}=1$.

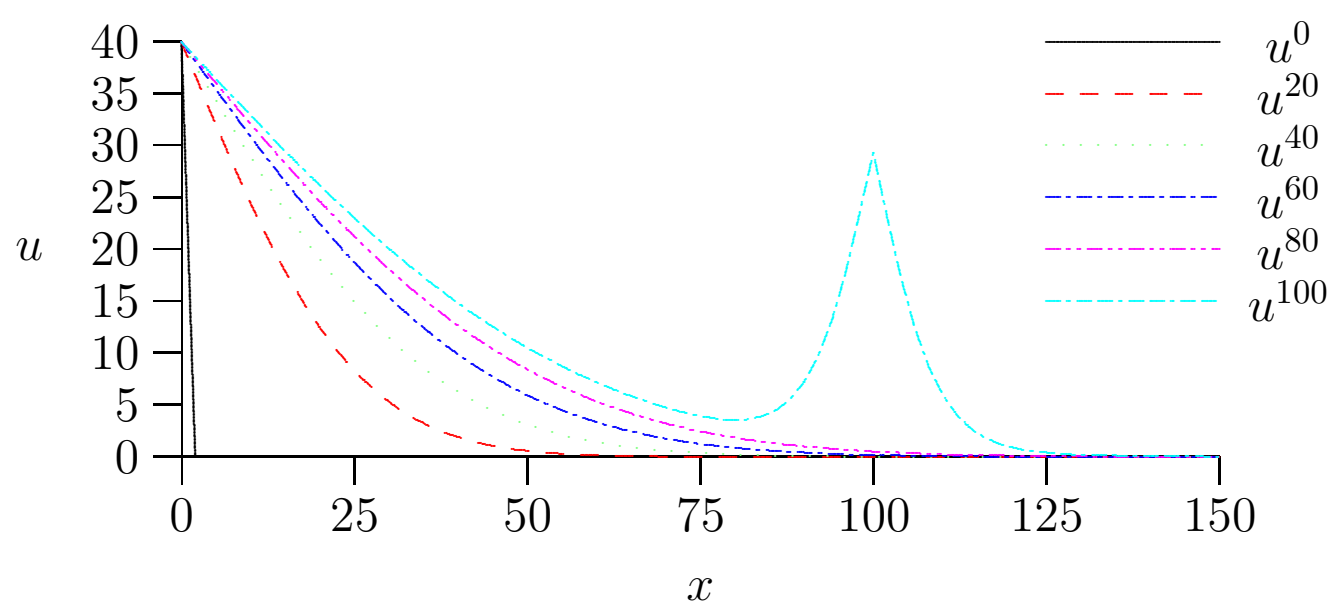

FIGURE 2. Evolution of $u_{i}^{j}$ in time and space and time $(\Delta x=1$, $\Delta t=0.5, r=2)$.

a erratic behavior of the solution for the largest values of $\Delta x$, producing negative cost (in these case, the value of $u$ exceeds $u_{b}$ ).

Figure 2 shows the evolution of $u_{i}^{j}$ in time and space.

\section{Conclusions}

We conclude that the injection is done in the beginning of the chamber, and near the final time (for the time dependent case). In what concerns the error, it is smaller in the time independent case. Finally, as expected, in what respect the cost, it is always small in the second case. 


\begin{tabular}{|c|c|c|c|c|c|c|c|c|}
\hline \multicolumn{9}{|c|}{$\underline{r}=2$} \\
\hline \multicolumn{3}{|c|}{ step } & \multicolumn{4}{|c|}{ optimal solution $\left(q_{i}^{j} \neq 0\right)$} & \multicolumn{2}{|c|}{ quality of the numerical solution } \\
\hline$\Delta t$ & $\Delta x$ & $j$ & $m-3$ & $m-2$ & $m-1$ & $m$ & cost & error $(\%)$ \\
\hline \multirow[t]{4}{*}{0.5} & 1 & $q_{1}^{j}$ & 104.7 & 238.6 & 243.2 & 113.5 & 116183.8 & 11.086 \\
\hline & 0.5 & $q_{1}^{j}$ & 98.4 & 236.7 & 247.1 & 117.8 & 143763.9 & 6.5928 \\
\hline & 0.25 & $q_{1}^{j}$ & 87.9 & 231.6 & 252.9 & 127.7 & 157705.3 & 2.9495 \\
\hline & 0.125 & $q_{1}^{j}$ & 67.7 & 220.8 & 263.7 & 147.8 & 164658.2 & - \\
\hline \multirow[t]{4}{*}{1} & 1 & $q_{1}^{j}$ & 54.2 & 120.8 & 120.8 & 54.2 & 132477.3 & 10.576 \\
\hline & 0.5 & $q_{1}^{j}$ & 53.9 & 121.1 & 121.1 & 53.9 & 152105.5 & 6.4065 \\
\hline & 0.25 & $q_{1}^{j}$ & 53.9 & 121.1 & 121.1 & 53.9 & 161966.8 & 3.0196 \\
\hline & 0.125 & $q_{1}^{j}$ & 53.9 & 121.1 & 121.1 & 53.9 & 166903.3 & - \\
\hline \multicolumn{9}{|c|}{$\underline{r=5}$} \\
\hline \multicolumn{3}{|c|}{ step } & \multicolumn{4}{|c|}{ optimal solution $\left(q_{i}^{j} \neq 0\right)$} & \multicolumn{2}{|c|}{ quality of the numerical solution } \\
\hline$\Delta t$ & $\Delta x$ & $j$ & $m-3$ & $m-2$ & $m-1$ & $m$ & cost & error $(\%)$ \\
\hline \multirow[t]{4}{*}{0.5} & 1 & $q_{1}^{j}$ & 93.9 & 232.9 & 248.9 & 124.3 & 83616.2 & 12.419 \\
\hline & 0.5 & $q_{1}^{j}$ & 74.6 & 224.1 & 259.8 & 141.5 & 111181.0 & 7.5516 \\
\hline & 0.25 & $q_{1}^{j}$ & 39.2 & 205.5 & 278.9 & 176.3 & 125073.8 & 3.6065 \\
\hline & 0.125 & $q_{1}^{j}$ & 0 & 180.3 & 300.0 & 219.7 & 131997.5 & - \\
\hline \multirow[t]{4}{*}{1} & 1 & $q_{1}^{j}$ & 53.3 & 120.5 & 121.0 & 54.7 & 100739.6 & 10.311 \\
\hline & 0.5 & $q_{1}^{j}$ & 4.8 & 94.8 & 147.4 & 103.0 & 120197.2 & 6.7237 \\
\hline & 0.25 & $q_{1}^{j}$ & 0 & 85.8 & 150.0 & 114.2 & 129948.5 & 3.1956 \\
\hline & 0.125 & $q_{1}^{j}$ & 0 & 71.5 & 150.1 & 128.3 & 134809.5 & - \\
\hline
\end{tabular}

TABLE 3. Results for time dependent $Q$ and $r$ equally distributed source terms in $[0, L]$.

\section{References}

[1] M. Bazaraa, H. Sherali and C. Shetty. Nonlinear Programming: Theory and Algorithms. John Wiley, New York, 1993.

[2] Geun-Cheol Gil, In-Seop Chang, Byung Hong Kim, Mia Kim, Jae-Kyung Jang, Hyung Soo Park, and Hyung Joo Kim. Operational parameters affecting the performance of a mediatorless microbial fuel cell. Biosensors and Bioelectronics, 18:327-334, 2003.

[3] S. Nash and A. Sofer. Linear and Nonlinear Programming. Mc. GrawHill, New York, 1996.

[4] J. Nocedal and S. Wright. Numerical Optimization. Springer, New York, 1999.

[5] R.S. Varga. Matrix Iterative Analysis. Prentice-Hall, New Jersey, 1962.

[6] Eric A. Zielke. Numerical analysis of a one dimensional diffusion equation for a single chamber microbial fuel cell using a linked simulation optimization (LSO) technique. Advanced Numerical Methods, E521, 2006.

[7] J.W. Thomas. Numerical Partial Differential Equations: Finite Difference Methods. SpringerVerlag, New York, 1995. 
OPTIMAL SOLUTION OF A DIFFUSION EQUATION WITH A DISCRETE SOURCE TERM 13

\begin{tabular}{ccccccccc} 
& & \multicolumn{7}{c}{$\Delta x$} \\
\cline { 3 - 8 }$\alpha$ & $\Delta t$ & & 4 & 2 & 1 & 0.5 & 0.25 & 0.125 \\
\hline \hline & & $v_{1}$ & 4 & 2 & 1 & 0.5 & 0.25 & 0.125 \\
& & $n$ & 77 & 155 & 310 & 620 & 1240 & 2480 \\
\hline 20 & 0.5 & cost & -127641.7 & -31037.8 & 14273.1 & 29597.4 & 33846.9 & 34854.3 \\
& & error (\%) & 13.8508 & 9.4618 & 3.5211 & 1.2170 & 0.3484 & - \\
\cline { 2 - 8 } & 1.0 & cost & -69297.5 & -1164.2 & 25064.6 & 34267.0 & 35041.8 & 35086.8 \\
& & error (\%) & 15.3702 & 6.5694 & 2.5745 & 0.0810 & 0.0982 & - \\
\hline 86.4 & 0.05 & cost & -217842.2 & -88258.8 & -10545.8 & 21231.2 & 31312.1 & 34099.5 \\
& & error (\%) & 15.1854 & 7.3978 & 4.4418 & 1.5628 & 0.4504 & - \\
\cline { 2 - 8 } & 0.5 & cost & -27291.6 & 15677.5 & 30011.4 & 33951.0 & 34876.0 & 35043.2 \\
& & error (\%) & 6.2560 & 2.3414 & 0.8387 & 0.2857 & 0.0813 & - \\
\cline { 2 - 8 } & 1.0 & cost & 1169.3 & 25818.1 & 34407.2 & 35051.1 & 35091.8 & 35099.1 \\
& & error (\%) & 4.3718 & 1.7369 & 0.0347 & 0.0210 & 0.0696 & - \\
\hline
\end{tabular}

TABLE 4 . Results for $v_{1}=1$.

\author{
A. Araújo \\ CMUC, Department of Mathematics, University of Coimbra, 3001-454 Coimbra, Portugal \\ E-mail address: alma@mat.uc.pt \\ Maria F. Patrício \\ CMUC, Department of Mathematics, University of Coimbra, 3001-454 Coimbra, Portugal \\ E-mail address: mfsp@mat.uc.pt \\ José L. SANTOS \\ CMUC, Department of Mathematics, University of Coimbra, 3001-454 Coimbra, Portugal \\ E-mail address: zeluis@mat.uc.pt
}

